UN IVERSITY OF COPENHAGEN

\title{
Extended Emotions
}

Krueger, Joel; Szanto, Thomas

Published in:

Philosophical Compass

DOI:

10.1111/phc3.12390

Publication date:

2016

Document version

Peer reviewed version

Citation for published version (APA):

Krueger, J., \& Szanto, T. (2016). Extended Emotions. Philosophical Compass, 11(12), 863-878.

https://doi.org/10.1111/phc3.12390 


\title{
Extended Emotions
}

\author{
Joel Krueger \& Thomas Szanto
}

\begin{abstract}
Until recently, philosophers and psychologists conceived of emotions as brain- and body-bound affairs. But researchers have started to challenge this internalist and individualist orthodoxy. A rapidly growing body of work suggests that some emotions incorporate external resources and thus extend beyond the neurophysiological confines of organisms; some even argue that emotions can be socially extended and shared by multiple agents. Call this the extended emotions thesis (ExE). In this article, we consider different ways of understanding ExE in philosophy, psychology, and the cognitive sciences. First, we outline the background of the debate and discuss different argumentative strategies for ExE. In particular, we distinguish ExE from cognate but more moderate claims about the embodied and situated nature of cognition and emotion (section 1). We then dwell upon two dimensions of ExE: emotions extended by material culture and by the social factors (section 2). We conclude by defending ExE against some objections (section 3) and point to desiderata for future research (section 4).
\end{abstract}

\section{Keywords}

Extended Mind Thesis; Embodiment; Situated Cognition; Extended and Distributed Emotions; Emotion Regulation; Collective Emotions

Defenders of the extended mind thesis (ExM) argue that some cognitive processes like thinking, planning, and navigating our environment extend beyond the head. Features of our environment drive and thus partially constitute some of these processes. Might emotions be similarly extended?

Until recently, most emotion researchers assumed that emotions supervene on processes inside an individual's brain and body. But some now question this internalist and individualist orthodoxy. An increasing number of researchers argue that some emotions incorporate external resources so deeply that they can be said to extend beyond brain and body; some even argue that emotions can be socially extended and shared by multiple agents. Call this the extended emotions thesis $(\mathrm{ExE})$. 
Recent decades have seen an increased philosophical interest in emotions. ExE can play an important role in broadening the scope of this renewed interest. While a relatively new area of research, ExE has already garnered much interest-in part because it picks up on themes that have been present in other disciplines such as social psychology and sociology for some time. Although its origins are philosophical, ExE is, as we'll see, poised to bring philosophy into contact with some lively interdisciplinary debates.

In what follows, we consider different ways of understanding ExE in philosophy and cognitive science. First, we do some background work and distinguish ExM and ExE from similar but more moderate claims about the embodied and situated nature of mind. Next, we look at different ways of arguing for ExM and ExE. We then consider two dimensions of ExE: emotions extended by features of material culture and the social world. We conclude by briefly considering some objections and implications for future research.

\section{From Embodied and Situated to Extended Emotions}

\section{Mind in body, mind in world}

In order to properly situate ExM and ExE in the philosophical landscape, note first that ExM and ExE are compatible with-but nevertheless distinct from - two other claims. These are claims about the embodied (Shapiro, 2011) and situated (Robbins and Ayedale, 2009) nature of mind.

From an embodied perspective, aspects of cognition and emotion depend on-and can be co-constituted by-not just brain processes but also bodily processes and the dynamics of our embodied agency. The embodied emotion thesis, in particular, is fueled by studies suggesting that emotional episodes are partially composed of perceptual, motoric, autonomic, and somatovisceral processes - a range of bodily components at work beyond the brain (for overviews, see Colombetti, 2014; Damasio, 1994; Davidson 2009; Kever et al 2016; Laird 2007; Niedenthal 2007). Recent debates have also focused on how emotional appraisal-at one time thought to be a distinctively cognitive component (Solomon 1976)—depends on extra-neural bodily processes (Barlassina and Newen 2013; Colombetti 2007; Maiese 2014).

From this perspective, one might say that emotions are bodily extended in that the vehicles of emotional phenomena span neural and extra-neural processes. But this is neither a new nor a philosophically controversial idea. In addition to many contemporary adherents, the embodied emotions thesis has received a great deal of 
support in the history of philosophy. (Colombetti and Thompson 2008; Stephan et al 2014). What's important to note, rather, is that the embodied emotions thesis is distinct from ExE. While it shares ExE's commitment to the claim that vehicles of emotion span neural and extra-neural components, the latter are nevertheless thought to be biological components wholly inside the individual.

A situated perspective is likewise in the vicinity of ExM and ExE although importantly distinct from it. From a situated perspective, cognition and emotion are inextricably embedded in rich networks of environmental (biological, sociocultural, material) scaffolding that make an ongoing contribution to their nature and functioning. Agents actively manipulate this scaffolding in order to boost thinking and intelligent action by reducing cognitive load (Clark 1995, pp.59-67; Kirsh 1995). A situated approach to emotions might characterize emotional displays as skillful and adaptive processes of modulating others' behavior by sending social signals designed to convey appraisals, elicit specific responses, and coordinate joint actions (Griffiths and Scarantino, 2009; Wiltusky 2015). Smiles, for example, are more often performed when interacting with others than when alone (Fernández-Dols et al. 1995).

From this perspective, the environment makes an active contribution to the performance of cognitive processes; it provides an ongoing flow of task-relevant information affecting both processing and responsive behavior. However, a situated perspective generally doesn't characterize the environment or the information it harbors as literally constituting part of the cognitive or emotional process. ${ }^{1}$ While sensitive to the complex dynamics interlacing mind and world, a situated perspective is nevertheless - like an embodied perspective-consistent with an individualistic and internalist view of cognition and emotions. ${ }^{2}$

\section{Mind outside the head: Arguing for ExM and ExE}

In contrast to embodied and situated perspectives, ExM and ExE argue that cognition and emotions can extend beyond brain and body. In these cases, parts of the world set up, drive, and regulate some cognitive and emotional processes and thus ought to be considered proper parts of these processes. There are generally said to be two waves of ExM arguments in the literature (Sutton 2010): The first involves the so-called 'parity principle' (PP); the second emphasizes the complementarity and

\footnotetext{
${ }_{1}$ Griffiths and Scarantino (2009: 448), for instance, are clear that their situated view does not commit them to an extended account of emotions.

2 See Stephan et al. (2014: 70-73) for further discussion of a situated or "embedded" approach to emotions and why it's distinct from ExE.
} 
integration (or "coupling") of internal and external processes as they form extended systems. ${ }^{3}$

First-wave PP approaches stress the functional similarity of internal and external processes. The idea is that:

If, as we confront some task, a part of the world functions as a process which, were it done in the head, we would have no hesitation in accepting as part of the cognitive process, then that part of the world is [...] part of the cognitive process. (Clark and Chalmers 1998: 8)

Clark and Chalmers provide several cases to motivate PP. The most famous involves Otto, an individual with memory loss due to Alzheimer's. Otto writes down all new information he acquires in his ever-present notebook, which he regularly uses to perform various cognitive tasks like remembering the location of MoMA. For PP, the notebook is part of the vehicle by which Otto realizes both his occurrent and his dispositional memories. It plays the same functional role that Otto's bio-memory does vis-à-vis his thought and behavior, and thus there's no principled reason for withholding it legitimate cognitive status.

Critics have attacked PP in a number of ways that need not concern us here (see sec. 3). What's more important is that these criticisms highlight an apparent weakness of PP in terms of how best to characterize the relationship between individuals and their cognitive artifacts. By focusing entirely on coarse-grained functional similarity, PP seems to overlook explanatorily significant structural and functional differences between internal and external resources. These differences are of particular interest to interdisciplinary approaches to mind, which are concerned with teasing out idiosyncratic effects different artifacts have on dimensions of our cognitive and affective life (Sutton 2010). Since ExE is motivated by interdisciplinary approaches sensitive to these differences, a conceptual tool other than PP is needed.

We find several tools in second wave "integration"-based ExM arguments (Menary, 2007; Rowlands, 1999; Sutton 2010). These arguments turn parity objections on their head by embracing idiosyncratic differences between internal and external resources. They're also concerned with exploring details of how users exploit and interact with these differences. From this integrationist perspective, the focus should be on clarifying how these differences work together (i.e., integrate) in driving intelligent thought, action, and emotion.

\footnotetext{
${ }^{3}$ Kirchhoff (2012) lays the groundwork for a third wave emphasizing the role that enculturation and patterned practices play in transforming the brain's representational capacities.
} 
To make this complementarity and integrationist view concrete, consider memory. As Merlin Donald (1991) argues, it's precisely because external memory devices within material culture- "exograms" like written records, measuring systems, and other artifacts—-work differently than biological modes of remembering that the former are poised to complement and integrate with the latter. For example, in contrast to internal bio-memories, external memories in notebooks or smartphone apps can be reformatted, are relatively permanent, have nearly-unlimited storage capacity, and offer multiple paths of access and retrieval. When users engage with these external devices, the integration of internal and external resources working together enables them to realize augmented forms of memory the brain simply can't achieve on its own. Similar benefits are can be found in the social world-for example, when individuals off-load some processes of recall onto others and groups collectively pool their resources to construct shared episodic memories (Harris et al 2014; Huebner 2016).

To return to ExE: as will become clear, the format and dynamics of external emotional resources matter. Different kinds of resources-whether found in material culture or the social world-have different properties, formats, and functions, and thus invite different forms of engagement, integration, and emotional enhancement.

\section{Types and Components of Emotions}

Proponents of ExE face at least two significant explanatory challenges. First, the realm of emotions and affectivity is very broad, from fleeting bodily feelings to longrunning moods or emotional dispositions. Despite this diversity, however, ExE proponents have so far failed to adequately distinguish claims about the possibility of extending various properties, components or types of different emotional and affective phenomena. A complete defense of ExE (beyond the scope of this discussion) thus requires not just a consideration of how paradigmatic emotional states like fear, anger, sadness, or joy might be extended, but also affective phenomena comprised of different components and temporal profiles: bodily affects, existential feelings, moods, temperaments, sentiments, character traits, dispositions, and the like. This is a tall order, one that has not yet been met in the ExE literature (although see Colombetti and Roberts 2015 and Stephan et al 2014 for some taxonomic considerations).

Second, ExE must also deal with the structural complexity of emotions. Emotions are multidimensional phenomena. And this structural complexity presents 
challenges in terms of specifying which parts of emotions are extended, when this occurs, and how integration with external resources realizes this extension.

How one begins to answer these questions turns on the model of emotions one accepts. According to Scherer's (2005) influential "component process model", emotions consist of five components: (i) an evaluative cognitive component (appraisal); (ii) a neurophysiological component (e.g., bodily symptoms); (iii) a motivational component (action tendencies); (iv) expressive motor components (e.g., facial and vocal expressions, mimicry); and (v) a subjective feeling component (experience). James Gross (2002, 2014) and others have recently argued that emotions are also constituted by a regulative component through which one initiates, inhibits, or modulates various components of an emotional episode.

Various attempts have been made to show how some of these different components might extend. For example, Krueger (2012) summons different sources of empirical support to argue that expressive components (facial expressions, gestures, etc.) constitute proper parts of some emotions and thus extend them beyond the head. Carter et al. (forthcoming) take a different tack and maintain that the most plausible candidate for extension is the non-conscious cognitive appraisal component. They argue that if some cognitive processes extend into the world-and some of these processes feature in appraisals that cognitivists like Nussbaum (2001) and Solomon (1988) argue are essential to emotions - then some emotions will be extended. However, this argument for $\mathrm{ExE}$ is based on cognitivist assumptions about emotions not shared by everybody in the debate (Scarantino, 2010).

Huebner (2011) similarly adopts a cognitivist and functionalist approach in arguing that emotions and moods can extend across collectives by the right sort of computational scaffolding. According to this view, emotions are essentially "tools for representing the world in particular ways" (113). The representational function of an emotion for Huebner is dissociable from its experiential aspect-and he argues further that there is no principled reason why such representational information (which may be non-conscious) cannot be socially distributed across individuals via technology or other means.

What about extending emotion's experiential component? Some proponents of ExM are wary of extended consciousness (e.g., Clark 2009; but see Ward 2012). Likewise, some otherwise friendly to ExE are nevertheless skeptical of extended emotional consciousness (Stephan et al. 2014; Sterelny 2010). However, this hesitation may rest on a tacit internalism about emotional experience: namely, as comprised of a detachable inside-the-head 'feeling' component functionally isolated 
from its other (potentially extended) components. But there may be reasons to resist this internalism by acknowledging an inextricable, mutually supporting relation between emotion's experiential, expressive, and regulative components (Frijda, 1986; Tomkins, 1984). An emerging trend in ExE literature (explored in more detail below) is, accordingly, to argue that the experiential dimension of emotions can be extended via external resources that scaffold these expressive and regulative dimensions.

With this background in place, we now turn to a more focused consideration of ExE. We consider two dimensions along which ExE proponents argue emotions might be extended: emotions extended by (1) material culture, and (2) socially extended emotions.

\section{Dimension of ExE}

\section{ExE and Material Culture}

There is a close connection between emotion regulation and material culture. ${ }^{4}$ In everyday life we manipulate artifacts and spaces in order to manipulate our emotional phenomenology. We play music, turn lights up or down, paint walls with specific colors, drink wine, burn incense, or wear specific items of clothing in order to evoke and sustain specific affective experiences (Colombetti and Krueger, 2015).

But not all of these manipulations count as extended emotions. After a long day at work, Inga might come home and light a few candles to create a soothing atmosphere. But the candles aren't necessarily part of Inga's ongoing emotional experience. The causal relation here is unidirectional: from world (candles) to Inga. Her response to the soothing qualities of the candlelight need not affect a material change in the environment. Accordingly, Inga hasn't integrated with the affectively salient properties of candles in any sense relevant to ExE. However, there are more philosophically interesting cases of emotion regulation that do seem to involve genuine subject-world integration-bi-directional engagements with material culture whereby external resources take over and govern regulative dynamics in an ongoing way.

From the perspective of ExE, these cases of materially-scaffolded regulations are good candidates for emotional extension. They are examples where a subject becomes part of what Wilson (2010) refers to as a functionally integrated, gainful system (hereafter, FIG). FIGs have three dimensions: they consist of processes that are (1)

\footnotetext{
${ }^{4}$ See Malafouris (2013) for a more general discussion of ExM and material culture.
} 
coupled, in that they are linked by reliable causal connections; (2) integrated, in that they are mutually-influencing and working together as one; and (3) functionally gainful, in that these processes together realize novel functions they can't realize separately.

Philosophers and musicologists alike argue that making music is a good example of a FIG (Borgo, 2011; Clark, 2008: 24; Cochrane, 2008; Colombetti and Roberts, 2015; Geeves and Sutton, 2015; Roberts, 2015). When a musician practices with her instrument, we might characterize this engagement as an ongoing process of emotional self-stimulation: physical qualities of the instrument and auditory properties of the produced sound (tempo, intensity, volume, etc.) coax specific emotional responses out of the listener and regulate the quality and dynamics of her experience in real-time-experiences that affect what she plays next, which regulate her further emotional experiences, etc. When actively integrated with her instrument, the musician may be able to realize emotional experiences with a particular intensity, depth, and diachronic character that are possible only when she is part of this materially-extended musical feedback loop. The physical basis of her experience is the activity realized in this feedback loop considered as a whole.

What about music listening? We routinely use music to regulate our emotions (Saarikallio 2011). Yet it might seem that music listening is a paradigm case of unidirectional (i.e., world to subject) causal relations. We play music and it impacts our emotional consciousness. But we don't affect any change in the music itself - an environmental manipulation needed to establish the integrative dimension of a FIG. However, a closer look at the sensorimotor dynamics coupling our listening experiences with our listening technologies shows things may not be this simple (Clarke, 2005; Krueger, 2014; Witek, 2013).

First, musical engagements are always mediated by material culture (DeNora, 2000). Music is materialized in technologies that afford ongoing manipulation. And these technologies are ultraportable: MP3 players and ubiquitous streaming services mean that listeners-like Otto and his notebook—can be coupled to their music as often and as long as they wish. The first dimension of a FIG is satisfied.

How might listener and music become actively integrated? The materiality of our music listening technologies means that we can manipulate music in real-time and, in so doing, manipulate our emotional experience (Skånland, 2013). We construct playlists and juxtapose artists, genres, and individual tracks, depending on our mood. We can even manipulate the auditory properties of the piece themselves (volume, treble, bass, etc.) within different listening contexts (headphones, speakers, etc.) in order to intentionally evoke a different array of emotional motor responses. 
The physical structure of our listening artifacts and their manipulations are part of our repertoire of everyday listening practices (Krause et al 2015).

Thinking of music as material culture thus highlights how music is something that can be manipulated in all sorts of user-specific ways-crucially, with a modulatory impact on our emotional experience. Our musical manipulations loop back onto us and shape our future manipulations and responsive behavior (Windsor and de Bézenac, 2012). Accordingly, it seems that, as with the musician and her instrument, these cases are similar instances of emotional self-stimulation. Via iterative cycles of motor entrainment and synchronization that unfold in response to musical dynamics, auditory properties of the music directly modulate a cascade of emotionspecific neural and physiological responses, coaxing emotions out of us and regulating their dynamics in real-time as they unfold over short and long-term listening episodes (Elvers 2016; Fritz el al., 2013; Lundqvist, 2009; Witek et al., 2014). And these musical feedback loops seed functional gain. When coupled with our musical artifacts, we gain access to self-regulatory and emotional capacities-for example, as Cochrane (2008) argues, an expanded phenomenological repertoire reflecting the expressive subtlety and richness of the music-that would remain otherwise inaccessible. Some argue that we are motivated to enact these musical couplings from birth (Haslbeck, 2014; Krueger, 2013a; Teckenberg-Jansson et al., 2011).

Several musicologists and philosophers defend a picture of music and ExM from a slightly different perspective. Kersten (2014), for example, draws on Gibson's ecological psychology to defend a view of music perception as an extended computational system (cf. Clarke, 2005; Matyja and Schhiavio, 2013). But these approaches, while helpful for allies of ExM, say little of musical emotions. Highlighting the relation between musical responsiveness and material culture, however, illuminates why music continues to be a potent cross-cultural resource for extending emotions - and it further clarifies why music has thus far been a central topic in ExE debates.

But defenders of ExE need not limit their analysis to music. There are other cases where processes of emotion regulation appear to be partially off-loaded onto the environment in that they loop through our ongoing interactions with different parts of our material culture: e.g., items of clothing and accessories like handbags (Kaufmann, 2011), religious artifacts (Krueger, forthcoming), the "affective atmospheres" of corporate spaces (Slaby, 2016) or music and dance guided therapy sessions (Maiese, 2016). Because the format and dynamics of these external resources are different than neural resources, they are poised to open up new modes of 
emotion experience and expression. And to characterize these extended regulatory processes as exclusively internal, ExE proponents suggest, is to ignore their dynamically decentered character-that is, the extent to which the individual's endogenous resources are no longer the sole locus of emotional control.

\section{ExE and the social world}

Others have turned to the social world to defend ExE. Discussions here concern the way emotions may be said to extend along two different levels of sociality: the faceto-face interpersonal level, as well as a more complex collective level involving group, intergroup, and habitualized sociocultural processes (Slaby, 2014; Parkinson et al., 2007).

Consider first the interpersonal versions of ExE, typically involving dyadic or face-to-face encounters between persons. Paradigm cases here are infant-caretaker interactions with close body contact. Because they lack endogenous mechanisms for attentional control, perceptual discrimination, or emotion regulation (e.g., regulating distress), young infants depend upon ongoing input from caregivers to realize cognitive and emotional function. This input is primarily provided not by material culture but by bodily interactions and vocal exchanges with caretakers. For example, a growing body of research has shown that by using different strategies to arouse, downplay or regulate the emotions of infants-smiling, vocal modifications, singing, caressing, diverting or guiding attention upon objects of distress, etc.-infants and caretakers tightly integrate their affective displays and realize emotional convergence (Krueger, 2013b). This is an instance of a co-regulatory process encompassing both infant and caretaker. Modes of emotional consciousness and self-regulatory function emerge between the two that are unique to this dyadic context (Taipale, 2016; Tronick, 1998). Consequently, social extensions of the expressive and regulative components of emotion can modulate or even determine the very way emotions 'feel like'.

This sort of interpersonally extended co-regulation, or "mutual affect", is not limited to infant-caregiver interactions but might also arise between two and more adults: a group of friends, romantic couples, or dance partners. For example, interpersonal off-loading of motor control in the rhythmic adaption to the movements of others in dancing can not only functionally enhance self-regulation but also generate emotional feedback loops on the fine-grained quality and depth of the very emotional experience of the involved subjects (Merritt, 2013). In some cases, they may trigger or even create genuinely new, emergent types or tokens of emotions (Varga \& Krueger, 2013; Krueger, 2014a; Varga, forthcoming) 
A much-discussed example of shared grief can illustrate this idea (Scheler, 1913/26; cf. Stephan et al., 2014; Schmid, 2014; Zahavi, 2015; Krueger, 2016; Szanto, forthcoming a; Léon, Szanto and Zahavi, forthcoming). The phenomenologists Max Scheler asks us to imagine a father and a mother standing together beside the dead body of their child. As they gaze at the child's corpse, both parents experience ongoing sequences of bodily-expressive feedback from the other: clenched muscles, held hands, the feel of the other's body wracked with quiet heaving, the gasps, sobs, and sounds of their mutual weeping, etc. Both partners have direct perceptual and tactile access to the grief of the other-expressions that in turn feed back onto, permeate, and directly modulate their own grief. Additionally, both partners share a common emotional focus (their dead child) and its deeply negative appraisal (sorrow) —as well as a common stock of shared memories, experiences, and associations that define the narrative history of their time with their child. The child's parents are thus affectivity integrated with one another at multiple levels and timesscales. For Scheler, this is a case of dyadically extended co-regulation that generates a shared emotion (grief) extending across both partners. Part of their experience of grief will be to feel the same emotion, toward the same object (their child), with the same or very similar intensity, and for the same reasons. Accordingly, they will experience their grief not as mine and your grief but rather as our grief-that is, as an interpersonally extended emotion.

Secondly, consider ExE on a group- or socio-cultural level. Since there is much confusion concerning these domains in the literature, often mixing up heterogeneous phenomena, it is useful to further distinguish between different mechanisms and types of affective extension:

(a) On the one hand, there are socio-psychological mechanisms pertaining to social identification and the psychological dynamics of intergroup relations. Consider how events concerning our own groups affect us personally and are of different emotional import than those concerning other groups. This may be because we are emotionally indifferent to other groups, or out-right hostile, thus establishing affective ingroup/out-group biases or divides (Parkinson et al., 2005). (b) On the other hand, social variants of ExE will involve also genuinely collective dimensions. The question here is whether emotions may be attributed not only along social boundaries and group divides but, rather, to collectives, corporations or nations as such. This research area builds on, but is different from earlier (Le Bon or Durkheim inspired) as well as contemporary social-psychological research on emotional contagion, automatic spreading of affects, their role for intra-group bonding, or crowd-dynamics such as mass hysteria (Hatfield et. al., 2014; Kelly et al., 2014). Moreover, the most interesting examples of also morally and politically relevant emotions have been 
discussed outside ExE research, in particular by sociologists (Stets and Turner, 2014), social psychologists (Smith et al., 2007), political philosophers and political scientists (Goodwin et al., 2001; Bar-Tal et al., 2007; Nussbaum, 2013). However, to re-assess these phenomena within ExE research would yield very interesting new results. Paradigm examples may include analyses of collective grief, e.g., in the wake of natural catastrophes, terrorism or other national traumata (Curci and Luminet, 2006), collective pride or self-esteem, such as patriotism, jubilatory emotions in the context of national sports events (Ehrenreich, 2007; Harth et al., 2008; Gilbert, 2009a; Nussbaum, 2013; Sullivan, 2014, 2015), and collective guilt or shame concerning genocide (Gilbert, 2002, 2009b; Sheehy, 2006; Harth et al., 2008).

Another way to differentiate within collective and socio-cultural scaffoldings concerns the different temporal and developmental dynamics of social extensions of emotions. On the one hand, there are synchronic processes that facilitate the performance and display of particular emotional episodes, such as scripts for certain rituals, social venues and sites (e.g., wedding 'scripts', musical arrangements, sports stadia, confessional boxes in a Catholic churches, socio-technological props like romantic web-platforms (Parkinson et al., 2005, 225ff.; Ben-Ze'ev, 2004; Illouz 2007 ), or the presence of peers. One should also mention here social appraisal and social referencing, i.e., processes by which one's own appraisal of an event is delegated or linked to others' appraisal (Klinnert et al., 2013). For example, children will typically find something disgusting if they recognize facial expressions of disgust by their caretakers.

On the other hand, there are diachronic elements scaffolding the development of emotional abilities and repertoires. Think of acquired and eventually internalized 'emotional norms' or 'feeling rules' ('Big boys don't cry') (Hochschild, 1983; von Scheve, 2012). Such social 'validation and legitimization' of emotions (Rimé, 2009) robustly shape the expression (or suppression) as well as the regulation of emotions. Moreover, they co-determine the salience of one's emotional target in the first place. In different socio-cultural contexts, different aspects of an object will affect me differently. Consider the way in which social norms, habits and conventions determine even such allegedly basic or universal and deeply embodied affective appraisals as disgust in the face of nutritional taboos (e.g., eating 'pets'). But examples of similarly robust socio-cultural scaffolding include also cultural institutions, such as the confession culture of the Catholic Church or psychoanalytic therapy. Illouz (2007) has proposed a useful conception in this context, namely a certain 'emotional habitus', i.e., internalized and internally structuring mechanism of managing one's own, and influencing others' emotions, which, again, are maintained by cultural, educational and socialization practices. Such scaffolding is typically fashioned by 
gender-roles, social status or role-conferred power and affects especially interpersonal emotions such as shame, envy or embarrassment. For instance, whether or not and why somebody is embarrassed or how one displays it, will largely depend on one's social status within a given social context or develop and adapt to different socio-cultural settings and particularly to responses of others (Goffman, 1956; Parkinson et al. 2005).

Current accounts of ExE primarily discuss the material culture scaffoldings involved on the interpersonal and the socio-cultural level of ExE (Colombetti and Krueger, 2015) or the technologically aided distribution of emotions across specialized teams, such as a navy-vessel navigation crew (Huebner 2011). For example, consider jointly attending to an object or event of emotional import with others, such as attending a concert. The fact that I am a co-attendant with others in the audience will modify how I perceive and aesthetically respond to the object or event, in part because others' reactive behavior and emotional responses may become constituents of my experience. The shared attentional framework in this context will, accordingly, co-regulate my individual emotional response. In other words, it may function as an external scaffolding expanding the complexity and character of my own experience insofar as other members of the audience provide regulatory input enabling me to access qualitative features of shared musical experiences that are otherwise inaccessible (Krueger, 2014a: 549-550; Cochrane 2009).

As this example again illustrates, linking the already large body of work on joint attention (e.g. Eilan et al., 2005; Seemann, 2011) and on co-regulation with ExE might significantly further research on each and a better understanding of the nature of socio-affective scaffolding and social forms of ExE (see León, Szanto, and Zahavi, forthcoming). Others stress the role of rather diffuse, emergent group-level phenomena such as 'emotional atmospheres' or 'climates' (Slaby, 2014; Stephan et al., 2014; cf. Rimé, 1991; Rivera and Páez, 2007). However, the social extension of emotions in terms of intergroup or social identification has remained unexplored within ExE research. Similarly, the constitutive function of robustly habitualized and distinctively normative components, such as previously-mentioned emotion norms, has been sidelined in the literature on ExE. Finally, much work remains to do in systematically linking debates within philosophy on socially extended and properly collective emotions (León, Szanto and Zahavi, forthcoming). Arguably, these directions represent fruitful new lines of research. Based on the pervious discussion, it seems that a central part of this research will involve a detailed analyses of the means, scaffoldings, and mechanism of extended co-regulatory processes. 


\section{Objections}

While ExM is an increasingly influential view in philosophy of mind, it's not without its critics. Many objections have been offered, some of which are relevant to ExE. We consider three: the 'coupling-constitution fallacy', the 'cognitive/emotional bloat' objection, and the 'mark of the cognition/emotions' objection.

As indicated, some remain neutral whether developmental and socio-cultural scaffoldings ought to be considered as instances of proper extensions or rather only as embedded or situated forms of emotions (Griffiths and Scarantino, 2009; Stephan et al., 2014). Echoing the alleged coupling-constitution fallacy (CCF) objection to ExM (Adams and Aizawa, 2008), the worry is that "an extended-emotion thesis potentially confuses the claim that the environment makes a causal contribution to a mental process with the more ontologically demanding claim that it is a constituent part of it" (Griffiths and Scarantino, 2009: 448).

In the face of such cautious neutralism, several points should be considered: first, many authors working within the ExM paradigm have already developed compelling responses to CCF (e.g., Clark, 2008, 2010; Kagan and Lassiter, 2013; Menary, 2010; Rowlands, 2010; cf. Krueger, 2012, 2014a). These responses are available to the ExE proponent. Additionally, the ExE proponent might say that $\mathrm{CCF}$ rests on a mischaracterization of their view. For, ExE does not claim that any environmental resource can become a constituent part of an emotion just because it makes a causal contribution to, or is coupled with, a particular emotional episode (recall the previous discussion of Inga and her candles). Rather, the concept of coupling is meant to specify when and why certain material or social resources are thus integrated with an affectively endowed agent such that they jointly fulfill a specific emotion-expressive or emotion-regulative function - to wit, functions that the agent would or could not perform without being appropriately coupled to those external resources.

This point might also be directed toward those otherwise sympathetic to ExE. Stephan and colleagues, for example, write that "without any additional criterion that distinguishes the 'interesting' couplings that give rise to integrated hybrid systems and real extension from those 'mere' couplings where external resources complement the cognitive machinery of a system without literally becoming a part of it, the integrationist's strategy therefore does not get off the ground" (Stephan et al. 2014, 76). ExE proponents might note that this does not license skeptics to dismiss any external props as potential candidates for coupling. Instead, it might be productive here to adopt Slaby's (2014) concept of “affective- 
phenomenal coupling", which seems broad enough to incorporate a variety of emotional scaffoldings, including other people, and yet as a heuristic concept is specific enough to indicate that ExE involves more than mere causal coupling.

That said, even those sympathetic to ExE should concede that the concept of coupling needs not only substantial disambiguation between causal dependency and functional integration but also between 'social integration' and 'phenomenal fusion' of subjects of experience (Schmid, 2009, 2014). For the latter task, one might go beyond ExM and ExE debates and look into the growing body of work in social ontology and social cognition research. Here we find resources to clarify the sense in which collective emotions involve a single, token-identical phenomenal subject of those emotions, i.e., some identification or fusion of emotional episodes of individuals - or, rather, the maintenance of a self/other-differentiation (Huebner, 2011; Helm, 2008, 2014; Salmela, 2012, 2014; Schmid, 2014; Szanto, 2015; Zahavi, 2015a, 2015b; Salmela and Nagatsu, 2016; Szanto, forthcoming a); Szanto, forthcoming b); León, Szanto, and Zahavi, forthcoming). Indeed, one of the central assumptions underlying skepticism regarding collective extensions of emotions is that either there is some ineffable phenomenal core to emotional experiences that is 'nontransferable' from one subject to another, or else that they must fuse into one tokenidentical emotional episode. As a possible reply to this defenders of ExE may first point to the inextricability of the different components of emotions, and especially their experiential, regulative and expressive components, the latter of which are often extra-organismic. Moreover, some have even contested that all emotions necessarily have an experiential feature, and that one can have emotions that are neither 'felt' nor conscious episodes (Roberts, 2003). Think of my anger at my boss that I realize to have had in the meeting yesterday only after I discuss the incident with my colleagues or my feeling proud of myself, because I cognitively assessed my achievement, but without any concomitant sensory, physiological or proper affective basis for that emotion. Such affective dissociations might even be more salient in collective cases and hence block that objection. A third strategy would be to refer to what social psychologists have aptly called the myth of the emotional artichoke (Averill and Nunley, 1992; Parkinson et al., 2007; cf. Goldie's (2000: 85, 99) “avocado pear conception of the emotions"). This is the view that whatever socio-cultural shells emotional experiences are clothed with, you may peel them away and eventually get to some allegedly non-social 'experiential core', the 'heart of emotions'. Rejecting this picture provides those who endorse ExE and especially the social extension of the experiential aspects of emotions further credit. 
Moreover, when it comes to genuinely shared emotions, mere causal influences will not suffice to differentiate these from emotional contagion (Hatfield et al., 2014), or similar social-psychological feedback dynamics. For example, reference to "contagious euphoria or panic of a crowd" (Stephan et al., 2012: 77), or a "contagious demagogue infecting the range of [one's] emotional repertoire" (Slaby 2014: 42), or the somewhat diffuse conceptions of "emotional atmospheres" and "climates" (de Rivera, 1992; Rimé, 2007) will rather hinder than help explaining what affective coupling really amounts to. By employing these examples-instead of what we might call synchronously or diachronically integrated 'emotional coalitions', such as romantic couples, caretaker-infant pairs, strongly cohesive minorities or groups constituted by some salient in-group/out-group affects (Yzerbyt et al., 2003; Seger et al., 2009)—one rather obstructs than facilitates to provide any clear-cut criterion for integration. Moreover, mere causal coupling of emotions, typical of emotional contagion and affective crowd dynamics, lack any robust integration of emotions. Emotional contagion may prevail without any explicit awareness of those feedbacks. It will typically occur without any awareness of emotional sharing, and is rather accompanied by a fusion or leveling of individual differences of expressive and regulative aspects of emotions.

Next, consider an analogous problem to the 'problem of cognitive bloat' or 'pancognitivism' (Adams and Aizawa, 2008, 2010; cf. Clark, 2008; Rowlands, 2010), which might be labeled the 'emotional bloat objection'. The concern is that if we engage in 'supersizing' the affective life of individuals, we may end up 'superdupersizing' it (Allen-Hermanson, 2013) and emotions may 'ooze' into everything and everybody and, conversely, everything may 'bleed' into the affective lives of individuals. However, the ExE proponent might once again respond that this worry is unwarranted once we specify the appropriateness of affective integration and delineate affectively deeper and more robust integrations. As discussed previously, there are a number of available routes here for the ExE defenderalthough there still lies some work ahead when it comes specifically to socially extended emotions.

Finally, consider what might be called the mark of the emotions objection and which mirrors the mark of cognition objection against ExM (Adams and Aizawa, 2008; cf. Rupert, 2009). According to this objection, we need an exact mark of cognition, one which is not derived from a priori conceptual analysis but rather provided by our best cognitive sciences; only such an empirical criterion will reliably distinguish entities that bear cognitive properties from those that are simply scaffolded by quasi-cognitive external props. The same might be required for 
emotions and one might then refer to developmental or social psychology or the affective neurosciences. However, the ExE defender might respond that it's question-begging to exclusively define emotions a priori in terms of some subjective (intra-)bodily feeling aspect. Moreover, they might also help themselves to a family of philosophical arguments that "emotions do not form a natural class" (Rorty, 1978: 141; cf. Barrett 2006 for further empirical evidence). Accordingly, from the perspective of ExE, this might be sufficient to show that there are no natural properties or prefixed facts that could determine whether something is or is not an emotion (see, critically, Roberts, 2003). And most agree that it is even less plausible to specify what a given emotion, say anger or sadness, is without reference to the respective sociocultural context. As Colombetti and Roberts argue, it seems that ExE can proceed with explanatorily relevant claims even in the absence of a "theorydriven 'mark of the affective"' (2015: 10).

\section{Conclusion and Future Research}

Discussions of ExE are still in their infancy-and metaphysical and empirical implications of ExE far from settled. However, what's already clear is that ExE offers a strong challenge to internalist and individualistic assumptions that, until recently, have shaped philosophical and psychological treatments of emotions. Philosophy of emotions is presently a lively area of research (see Goldie 2010). ExE can potentially help move these debates forward by offering a fresh critical perspective on the ontology of emotions.

As mentioned previously, future work on ExE should address pressing taxonomic considerations. This will involve not only specifying vehicles of emotional extension (material, social, cultural, etc.), but also specifying which components of emotions (cognitive, experiential, etc.) and which types of emotions (moods, temperaments, bodily affects, etc.) might or might not be extended by these different vehicles. In particular, discussing the hitherto understudied role of cultural artifacts in scaffolding emotions, such as individual and shared narratives, collective memory and imagination, or the role of shared habits and norms in shaping so-called 'emotional cultures', will greatly enrich current debates.

In light of its interdisciplinary approach, ExE is also poised to fruitfully supplement research in areas beyond philosophy. One such area is psychiatry and psychopathology. Drayson (2009) and Fuchs (2009) note that the recent embodied turn in philosophy of mind and cognitive offers ample theoretical resources for furthering our understanding of mental disorder. In particular, embodied cognitive 
science seems to challenge the metaphysical and epistemic reductionism behind the dominant biomedical model, according to which psychiatric disorders are best conceptualized (and treated) as brain disorders (Zachar, 2000). Some initial attempts have been made to indicate how ExM might impact issues of psychiatric taxonomy, diagnosis, and treatment (Davies, 2016; Sprevak, 2011). Instead of looking for the cause of mental disorders in specific brain regions or functions, ExM may instead compel us to see major psychiatric disorders-including the emotional disruptions characteristic of these disorders - as resulting from multidimensional breakdowns of the extended organism-environment loop. From the perspective of ExE and ExM, then, psychiatry would need to become an "ecological" enterprise in order to better grasp the interconnection of psychological, social, and pharmacological approaches adequate for its subject (Fuchs 2009: 232). ExM and ExE can make conceptual contributions necessary for developing such an ecological turn in psychiatry.

ExE research might further broaden its impact by developing more overt political and cultural studies dimensions (Protevi, 2009). At present, work exploring prevalent negative, disrupting, and politically relevant phenomena in connection with socially extended emotions is still scarce. For instance, think of the pervasive and more often than not negative and intruding effects that corporate, organizational, or workplace scaffoldings have on regulating individual and group emotions. Using an apt metaphor, Slaby (2016) speaks of the "hacking" of individuals' affective lives by corporate agents, who often frame and modulate individuals' affective lives without their consent. Further potentially fruitful investigations in this context might concern collaborative or organizational forms of irrationality and corresponding disruptions of emotional co-regulation. Here, too, various social scaffoldings are crucially contributing to these disruptions. For example, socio-psychological mechanisms such as the infamous groupthink (Janis, 1982), group polarization or choice shift in deliberative groups (Friedkin, 1999; Sunstein, 2002) or propaganda typically play a constitutive role in entering or maintaining practical irrationality, self-deception, or loss of affective control. And this, in turn, is often due precisely to their disruptive role on co-regulatory practices (misidentification of one's own or one's group's shared emotions, biases on one's own or a group's affect control, etc.) (see Szanto, forthcoming b.). Here, placing ExE in dialogue with political philosophy, social choice and game theory, and recent sociology of emotions is likely to generate genuinely new insights and open up new areas for further research. ${ }^{5}$ ${ }^{5}$ Michele Merritt has recently done related work on ExM and ExE in the context of gender (Merritt 2014)
and eating disorder (Merritt forthcoming). 


\section{References}

Adams, Fred, and Aizawa, Ken. The Bounds of Cognition. London: Wiley-Blackwell, 2008.

Adams, Fred, and Aizawa, Ken. 'Defending the bounds of cognition.' The Extended Mind. Ed. Richard Menary. Cambridge, MA: MIT Press, 2010. 66-99.

Allen-Hermanson, Sean. 'Superdupersizing the mind: Extended cognition and the persistence of cognitive bloat.' Philosophical Studies, 164.3 (2013): 791-806.

Averill, James R., Nunley, Elma P. Voyages of the heart: Living an emotionally creative life. New York: Free Press, 1992.

Barlassina, Luca, and Albert Newen. 'The Role of Bodily Perception in Emotion: In Defense of an Impure Somatic Theory.' Philosophy and Phenomenological Research, 89.3 (2014): 637-78.

Bar-Tal, Daniel, Eran Halperin, and Joseph De Rivera. 'Collective emotions in conflict situations: Societal implications.' Journal of Social Issues, 63.2 (2007): 441-60.

Barrett, Lisa Feldman. 'Are emotions natural kinds?' Perspectives on psychological science, 1.1 (2006): 28-58.

Ben-Ze'ev, Aaron. The Subtlety of Emotions. Cambridge, MA: MIT Press, 2000.

Borgo, David. 'The Ghost in the Music: Improvisers, Technology, and the Extended Mind.' Society for Electro-Acoustic Music, University of Miami. 2011.

Damasio, Antonio. Descartes' Error. Emotion, Reason, and the Human Brain. London: Penguin, 1994.

Carter, Adam J., Gordon, Emma C., and Palermos, Orestis S. 'Extended Emotion.' Philosophical Psychology, forthcoming.

Clark, Andy. Being There: Putting, Brain, Body, and World Together Again. Cambridge, MA: MIT Press, 1997.

Clark, Andy. Supersizing the Mind. Embodiment, Action and Cognitive Extension. Oxford: Oxford UP, 2008.

Clark, Andy. "Spreading the Joy? Why the Machinery of Consciousness Is (Probably) Still in the Head." Mind 118.472 (2009): 963-993.

Clark, Andy. 'Coupling, Constitution and the Cognitive Kind. A Reply to Adams and Aizawa.' The Extended Mind. Ed. Richard Menary. Cambridge, MA: MIT Press, 2010. 81-100.

Clark, Andy, and Chalmers, David. 'The Extended Mind.' Analysis 58.1 (1998): 7-19.

Clarke, Eric F. Ways of Listening: An Ecological Approach to the Perception of Musical Meaning. Oxford: Oxford UP, 2005.

Cochrane, Tom. 'Joint Attention to Music.' British Journal of Aesthetics 49.1 (2009): 59-73.

Collins, Randall. Interaction Ritual Chains. Princeton: Princeton UP, 2004.

Colombetti, Giovanna. 'Enactive Appraisal.' Phenomenology and the Cognitive Sciences 6.4 (2007): 527-46.

Colombetti, Giovanna (2014). The Feeling Body. Affective Science Meets the Enactive Mind. Cambridge, MA: MIT Press. 
Colombetti, Giovanna. 'Enactive Affectivity, Extended.' Topoi, forthcoming. doi: 10.1007/s11245-015-9335-2.

Colombetti, Giovanna, and Krueger, Joel. 'Scaffoldings of the Affective Mind.' Philosophical Psychology 28.8 (2015): 1157-1176.

Colombetti, Giovanna, and Roberts, Tom (2015). 'Extending the extended mind: the case for extended affectivity.' Philosophical Studies, 172.5: 1243-63.

Colombetti, Giovanna, and Thompson, Evan. 'The feeling body: toward an enactive approach to emotion', in: Overton, W.F., Müller, U., Newman, J. (Eds.), Developmental Perspectives on Embodiment and Consciousness. Lawrence Erlbaum, New York, 2008: 45-68.

Curci, Antonietta, and Olivier Luminet. 'Follow-up of a cross-national comparison on flashbulb and event memory for the September 11th attacks.' Memory, 14.3 (2006): 329-44.

Davies, Will. 'Externalist Psychiatry.' Analysis 76.3 (2016): 290-96.

DeNora, Tia. Music in everyday life. Cambridge: Cambridge University Press, 2000.

de Rivera, Joseph. 'Emotional climate: Social structure and emotional dynamics.' International Review of Studies on Emotion, Ed. K.T. Strongman. Vol. 2, London: Wiley, 1992. 197218.

de Rivera, Joseph, and Páez, Darío. 'Emotional Climate, Human Security, and Cultures of Peace.' Journal of Social Issues, 63.2 (2007): 233-253.

Drayson, Zoe. 'Embodied Cognitive Science and Its Implications for Psychopathology.' Philosophy, Psychiatry, \& Psychology: PPP 16.4 (2009): 329-40.

Ehrenreich, Barbara. Dancing in the streets: A bistory of collective joy. Macmillan, 2007.

El Kaliouby, Rana, Picard, Rosalind, and Baron-Cohen, Simon. 'Affective computing and autism.' Annals of the New York. Academy of Sciences, 1093.1 (2006): 228-48.

Elvers, Paul. 'Songs for the Ego: Theorizing Musical Self-Enhancement.' Frontiers in Psychology 7.2 (2016): 1-11.

Frijda, Nico H. The Emotions. Cambridge: Cambridge UP, 1986.

Fritz, Thomas H., Hardikar, Samyogita, Demoucron, Matthias, Niessen, Margot, Demey, Michiel, Giot, Olivier, Li, Yongmind, Haynes, john-Dylan, Villringer, Arno, Leman, Marc. 'Musical agency reduces perceived exertion during strenuous physical performance.' Proceedings of the National Academy of Sciences, 110.44 (2013): 17784-89.

Fuchs, Thomas. 'Embodied Cognitive Neuroscience and Its Consequences for Psychiatry.' Poiesis \& Praxis: International Journal of Ethics of Science and Technology Assessment 6.3-4 (2009): 219-33.

Geeves, Andrew and Sutton, John. 'Embodied Cognition, Perception, and Performance in Music.' Empirical Musicology Review, 9.3-4 (2015): 247-53.

Goodwin, Jeff, Jasper, James M., and Polletta, Francesca (Eds.). Passionate Politics: Emotions and Social Movements. Chicago: University of Chicago Press, 2001.

Goldie, Peter. The Emotions. A Philosophical Exploration. Oxford: Oxford UP, 2000.

Goldie, Peter., ed. The Oxford Handbook of Philosophy of Emotion. Oxford: Oxford UP, 2010. 
Griffiths, Paul E., and Scarantino, Andrea. 'Emotions in the wild: The situated perspective on emotion.' The Cambridge Handbook Of Situated Cognition. Ed. Philip Robbins, and Murat Aydede. Cambridge: Cambridge UP, 2009. 437-53.

Gross, James J. 'Emotion Regulation: Affective, Cognitive, and Social Consequences.' Psychophysiology, 39.3 (2002): 281-291.

Gross, James J., ed. Handbook of Emotion Regulation. Second Edition. New York: The Guilford Press, 2014.

Fernández-Dols, José-Miguel, and Ruiz-Belda, María-Angeles. 'Are Smiles a Sign of Happiness? Gold Medal Winners at the Olympic Games.' Journal of Personality and Social Psychology 69.6 (1995): 1113-19.

Friedkin, Noah E. 'Choice Shift and Group Polarization.' American Sociological Review, 64.6 (1999): 856-75.

Gilbert, Margaret. 'Collective guilt and collective guilt feelings.' The Journal of Ethics, 6.2 (2002): 115-43.

Gilbert, Margaret: 'Pro Patria: An Essay on Patriotism.' The Journal of Ethics, 13.4 (2009a): 319-46.

Gilbert, Margaret. 'Real Unity of Them All.' The Monist 92.2 (2009b): 268-85.

Gilbert, Margaret. 'How we feel: Understanding everyday collective emotion ascription.' Collective emotions. Perspectives from Psychology, and Sociology. Ed. Christian von Scheve, and Mikko Salmela. Oxford: Oxford UP, 2014. 17-31.

Harris, Celia B., Amanda J. Barnier, John Sutton, and Paul G. Keil. "Couples as Socially Distributed Cognitive Systems: Remembering in Everyday Social and Material Contexts." Memory Studies 7.3 (2014): 285-97.

Harth, Nicole Syringa, Kessler, Thomas, and Leach, Colin Wayne. 'Advantaged group's emotional reactions to intergroup inequality: The dynamics of pride, guilt, and sympathy.' Personality and Social Psychology Bulletin 34.1 (2008): 115-29.

Haslbeck, Friederike B. 'The interactive potential of creative music therapy with premature infants and their parents: A qualitative analysis.' Nordic Journal of Music Therapy, 23.1 (2014): 36-70.

Helm, Bennett. 'Plural Agents.' Nô̂s 42.1 (2008): 17-49.

Helm, Bennett. 'Emotional communities of respect.' Collective emotions. Perspectives from Psychology, and Sociology. Ed. Christian von Scheve, and Mikko Salmela. Oxford: Oxford UP, 2014. 47-60.

Hatfield, Elaine, Carpenter, Megan, and Rapson, Richard L. 'Emotional contagion as a precursor to collective emotions.' Collective emotions. Perspectives from Psychology, and Sociology. Ed. Christian von Scheve, and Mikko Salmela. Oxford: Oxford UP, 2014. 108-22.

Hochschild, Arlie. 'Emotion Work, Feeling Rules, and Social Structure.' American Journal of Sociology, 85.3 (1979): 551-75.

Huebner, Bryce. 'Genuinely collective emotions.' European Journal for Philosophy of Science, 1.1 (2011): 89-118. 
Huebner, Bryce. 'Transactive Memory Reconstructed: Rethinking Wegner's Research Program.' The Southern Journal of Philosophy 54.1 (2016): 48-69.

Illouz, Eva. Cold Intimacies. The Making of Emotional Capitalism. Cambridge: Polity, 2007.

Janis, Irvin L. Groupthink. Boston: Wadsworth, 1982.

Kagan, Aaron, and Lassiter, Charles. 'The coupling-constitution fallacy: Much ado about nothing.' Pragmatics \& Cognition 21.1 (2013): 178-92.

Kaufmann, Jean-Claude. Le sac : Un petit monde d'amour. Paris: Jean-Claude Lattès. 2011.

Kelly, Janice R., Iannone, Nicole E., and McCarty, Megan K. 'The function of shared affect in groups.' Collective emotions. Perspectives from Psychology, and Sociology. Ed. Christian von Scheve, and Mikko Salmela. Oxford: Oxford UP, 2014: 175-188.

Kersten, Luke. 'Music and Cognitive Extension.' Empirical Musicology Review: EMR 9 (3-4): 193-202. 2014

Kever, Anne, Delphine Grynberg, Marie Bayot, and Nicolas Vermeulen. "Embodying Emotions: The Role of Bodily Changes in Emotional Processing in Normal and Psychopathological Conditions." In Foundations of Embodied Cognition Volume 1: Perceptual and Emotional Embodiment, edited by Yann Coello and Martin H. Fischer. London and New York: Routledge, 2015: 246-261.

Kieverstein, Julian, and Farina, Mirko. 'Do sensory substitution devices extend the conscious mind?' Consciousness in Interaction: The role of the natural and social context in shaping consciousness. Ed. Fabio Paglieri. Amsterdam: John Benjamins, 2012. 19-40.

Kirchhoff, Michael David. "Extended Cognition and Fixed Properties: Steps to a ThirdWave Version of Extended Cognition." Phenomenology and the Cognitive Sciences 11.2 (2012): 287-308.

Kirsh, David. 'The intelligent use of space.' Artificial intelligence 73.1 (1995): 31-68.

Klinnert, Mary D., Emde, Robert N., Butterfield, Perry, and Campos, Joseph J. (1986). 'Social referencing: The infant's use of emotional signals from a friendly adult with mother present.' Developmental Psychology, 22.4 (1986): 427-32.

Krueger, Joel. 'Seeing mind in action.' Phenomenology and the Cognitive Sciences 11.2 (2012): 14973.

Krueger, Joel. 'Empathy, Enaction, and Shared Musical Experience: Evidence from Infant Cognition. The Emotional Power of Music: Multidisciplinary Perspectives on Musical Expression, Arousal, and Social Control. Ed. Thomas Cochrane, Bernardo Fantini, and Klaus Scherer. Oxford: Oxford University Press, 2013a. 177-196.

Krueger, Joel. 'Merleau-Ponty on shared emotions and the joint ownership thesis.' Continental Philosophy Review, 46.4 (2013b): 509-31.

Krueger, Joel. 'Varieties of extended emotions.' Phenomenology and the Cognitive Sciences, 13.4 (2014a): 533-55.

Krueger, Joel. 'Affordances and the musically extended mind.' Frontiers in Psychology, 4.1003 (2014b), 1-13. 
Krueger, Joel. 'Emotions and the Social Niche.' Collective emotions. Perspectives from Psychology, and Sociology. Ed. Christian von Scheve, and Mikko Salmela. Oxford: Oxford UP, 2014c. 156-71.

Krueger, Joel. 'Musical manipulations and the emotionally extended mind.' Empirical Musicology Review, 9.3-4 (2014d): 208-12.

Krueger, Joel. 'The Affective 'We': Self-regulation and Shared Emotions.' The Phenomenology of Sociality: Discovering the ' $W e$ '. Ed. Thomas Szanto, and Dermot Moran. London, New York: Routledge, 2016. 263-77.

Krause, Amanda E., Adrian C. North, and Lauren Y. Hewitt. 'Music-Listening in Everyday Life: Devices and Choice.' Psychology of Music 43.2 (2015): 155-70.

Laird, James D. Feelings: The Perception of Self. Oxford: Oxford UP, 2007.

León, Felipe, Szanto, Thomas, and Zahavi, Dan. 'Emotional Sharing and the Extended Mind.' Forthcoming.

Loughlin, Victor. 'Sketch This: Extended Mind and Consciousness Extension.' Phenomenology and the Cognitive Sciences, 12.1 (2013): 41-50.

Lundqvist, Lars-Olav, Carlsson, Fredrik, Hilmersson, Per, and Juslin, Patrick N. 'Emotional responses to music: experience, expression, and physiology.' Psychology of Music, 37.1. (2009): 61-90.

Maiese, Michelle. 'How Can Emotions Be Both Cognitive and Bodily?' Phenomenology and the Cognitive Sciences 13.4 (2014), 513-531.

Maiese, Michelle. 'Affective Scaffolds, Expressive Arts, and Cognition.' Frontiers in Psychology 7.359 (2016): 1-11.

Malafouris, Lambros. How Things Shape the Mind. Cambridge, MA: MIT Press, 2013.

Manstead, Anthony S., and Fischer, Agneta H. 'Social appraisal: The social world as object of and influence on appraisal processes.' Appraisal processes in emotion: Theory, research, application. Ed. Klaus Scherer, Angela Schorr, and Tom Johnstone. Oxford: Oxford UP, 2001. 221-232.

Matyja, Jakub Ryszard, and Andrea Schiavio. 'Enactive Music Cognition: Background and Research Themes.' Constructivist Foundations, 8.3 (2013): 351-57.

Menary, Richard. Cognitive Integration: Mind and Cognition Unbounded: Attacking the Bounds of Cognition. Basingstoke: Palgrave Macmillan, 2007.

Menary, Richard. 'Cognitive Integration and the Extended Mind.' Richard Menary (Ed.). The Extended Mind. Cambridge, MA: MIT Press, 2010. 227-43.

Menary, Richard (Ed.). The Extended Mind. Cambridge, MA, London: MIT Press, 2010.

Merritt, Michele. 'Thinking-Is-Moving: Dance, Agency, and a Radically Enactive Mind.' Phenomenology and the Cognitive Sciences 14 (1). Springer Netherlands: 95-110, 2013.

Merritt, Michele. 'Making (non)sense of Gender.' In Enactive Cognition at the Edge of Sense-Making: Making Sense of Non-Sense, edited by Massimiliano Cappuccio and Tom Froese, 285-306. New York: Palgrave Macmillan, 2014.

Merritt, Michele. 'Any Body Not My Own: Collective Emotions and the Constitution of Eating Disorders'. Frontiers in Psychology, Forthcoming. 
Niedenthal, Paula M. 'Embodying emotion.' Science, 316.5827 (2007): 1002-05.

Nussbaum, Martha C. Upheavals of thought: The intelligence of emotions. Cambridge: Cambridge UP, 2001.

Nussbaum, Martha C. Political Emotions. Why Love Matters for Justice. Cambridge, MA: Harvard UP, 2013.

Oatley, Keith. Best laid schemes: The psychology of the emotions. Cambridge: Cambridge UP, 1992.

Oatley, Keith. 'The sentiments and beliefs of distributed cognition.' Emotions and beliefs: How feelings influence thoughts. Ed. Nico H. Frijda, Anthony S.R. Manstead, and Sacha Bem (Eds.). Cambridge: Cambridge UP, 2000. 78-107.

Parkinson, Brian, Fischer, Agneta H., Manstead, Anthony S.R. (2005). Emotion in Social Relations. Cultural, Group, and Interpersonal Processes. New York: Psychology Press.

Picard, Rosalind W., and Healey, Jennifer. 'Affective wearables.' Personal Technologies, 1.4 (1997): 231-40.

Ping, Raedy M., Dhillon, Sonica, and Beilock, Sian L. 'Reach for what you like: The body's role in shaping preferences.' Emotion Review, 1.2 (2009): 140-50.

Protevi, John. Political Affect: Connecting the Social and the Somatic. Posthumanities Series. University of Minnesota Press. 2009.

Rimé, Bernard. 'The Social Sharing of Emotion as an Interface Between Individual and Collective Processes in the Construction of Emotional Climates.' Journal of Social Issues, 63.2 (2007), 307-322.

Rimé, Bernard. 'Emotion elicits the social sharing of emotion: Theory and empirical review.' Emotion Review, 1.1 (2009): 60-85.

Robbins, Philip, and Aydede, Murat (Eds.). The Cambridge Handbook of Situated Cognition. Cambridge: Cambridge UP, 2009.

Roberts, Robert C. Emotions: An essay in aid of moral psychology. Cambridge: Cambridge UP, 2003.

Roberts, Tom. 'Extending Emotional Consciousness.' Journal of Consciousness Studies, 22.3-4 (2015): 108-128.

Robinson, Douglas. Feeling Extended. Sociality as Extended Body-Becoming-Mind. Cambridge, MA: MIT Press, 2013.

Rorty, Amelie Oksenberg 'Explaining emotions.' The Journal of Philosophy, 75.3 (1978): 139_ 61.

Rowlands, Mark. The New Sciences of the Mind. From Extended Mind to Embodied Phenomenology. Cambridge, MA: MIT Press, 2010.

Russell, James A., and Feldman, Barrett, L. 'Core affect, prototypical emotional episodes, and other things called emotion: dissecting the elephant.' Journal of personality and social psychology, 76.5 (1999): 805-19.

Rupert, Robert D. Cognitive Systems and the Extended Mind. Oxford: Oxford UP, 2009.

Saarikallio, S. "Music as Emotional Self-Regulation throughout Adulthood." Psychology of Music 39 (3): 307-27. 2011. 
Salmela, Mikko. 'Shared emotions.' Philosophical Explorations, 15.1 (2012): 33-46.

Salmela, Mikko. 'The Functions of Collective Emotions in Social Groups.' Institutions, Emotions, and Group Agents. Anita Konzelmann Ziv, \& Hans Bernhard Schmid (Eds.), Dordrecht: Springer, 2014. 159-76.

Salmela, Mikko, and Nagatsu, Michiru. 'Collective Emotions and Joint Action. Beyond Received and Minimalist Approaches.' Journal of Social Ontology, 2.1 (2016): 33-57.

Scarantino, Andrea. 'Insights and blindspots of the cognitivist theory of emotions.' The British Journal for the Philosophy of Science, 61.4 (2010): 729-68.

Scherer, Klaus R. 'What are emotions? And how can they be measured?' Social science information, 44.4 (2005): 695-729.

Schmid, Hans Bernhard. 'The feeling of being a group: corporate emotions and collective consciousness.' Collective emotions. Perspectives from Psychology, and Sociology. Ed. Christian von Scheve, and Mikko Salmela. Oxford: Oxford UP, 2014. 3-16.

Schmid, Hans Bernhard. Plural Action. Essays in Philosophy and Social Science. Dordrecht: Springer, 2009.

Scheler, Max. Wesen und Formen der Sympathie. Gesammelte Werke, Vol. 7., Bonn: Bouvier 2005 (1912/1926). [The nature of sympathy. Trans. by P. Heath. London: Routledge \& Kegan Paul 1954.]

Seger, Charles R., Eliot R. Smith, and Diane M. Mackie. 'Subtle activation of a social categorization triggers group-level emotions.' Journal of Experimental Social Psychology, 45.3 (2009): 460- 67.

Shapiro, Lawrence A. Embodied Cognition. New York: Routledge, 2011.

Sheehy, Paul. 'Holding them responsible.' Midwest studies in philosophy, 30.1 (2006): 74-93.

Skånland, Marie Strand. 'Everyday Music Listening and Affect Regulation: The Role of MP3 Players.' International Journal of Qualitative Studies on Health and Well-Being, 8.20595 (2013): $1-10$.

Slaby, Jan. 'Emotions and the extended mind.' Collective emotions. Perspectives from Psychology, and Sociology. Ed. Christian von Scheve, and Mikko Salmela. Oxford: Oxford UP, 2014. 32-46.

Slaby, Jan. 'Mind Invasion: Situated Affectivity and the Corporate Life Hack.' Frontiers of Psychology, 7 (2016): 266. doi: 10.3389/fpsyg.2016.00266

Smith, Eliot R., Seger, Charles R., and Mackie, Diane M. 'Can emotions be truly group level? Evidence regarding four conceptual criteria.' Journal of personality and social psychology, 93.3 (2007): 431-46.

Solomon, Robert. The passions: Emotions and the meaning of life. Anchor Press, New York, 1976.

Solomon, Robert. 'On Emotions as Judgments.' American Philosophical Quarterly, 25.2 (1988): 183-91.

Sprevak, Mark. 'Neural Sufficiency, Reductionism, and Cognitive Neuropsychiatry." Philosophy, Psychiatry, \& Psychology, 18 (4): 339-44. 2011.

Stephan, Achim, Walter, Sven, and Wilutzky, Wilhelm. Emotions beyond brain and body. Philosophical Psychology, 27.1 (2014): 65-81. 
Stephan, Achim, and Walter, Sven. 'The Situated Perspective on Emotions: A Philosophical Roadmap.' Online: http://emotionresearcher.com/the-situated-perspective-onemotions-a-philosophical-roadmap/. 2015.

Stets, Jan E., and Turner, Jonathan H. (Eds.). Handbook of the Sociology of Emotions, Vol. II. Dordrecht: Springer, 2014.

Sullivan, Gavin Brent. 'Collective emotions.' Social and Personality Psychology Compass, 9.8 (2015): 383-93.

Sullivan, Gavin Brent. Understanding Collective Pride and Group Identity: New directions in emotion theory, research and practice. London, New York: Routledge, 2014.

Sutton, John. 'Exograms and Interdisciplinarity: History, the Extended Mind and the Civilizing Process.' The Extended Mind. Ed. Richard Menary. Cambridge, MA: MIT Press, 2010. 189-225.

Sunstein, Cass R. 'The law of group polarization.' The Journal of Political Philosophy 10.2 (2002): 175-95.

Szanto, Thomas. 'Collective emotions, normativity, and empathy: A Steinian account.' Human Studies 38.4 (2015): 503-27.

Szanto, Thomas (forthcoming a). 'The Phenomenology of Collective Emotions: A Critical Comparison of Walther's and Stein's Contributions.' Forthcoming in: Woman Phenomenologists on Social Ontology. Luft, \& R. Hagengruber (Eds.). Dordrecht: Springer.

Szanto, Thomas (forthcoming b). 'Collective Irrationality, Akrasia and Groupthink: Social Disruptions of Emotion Regulation.'

Taipale, Joona. 'Self-Regulation and Beyond: Affect Regulation and the Infant-Caregiver Dyad.' Frontiers in Psychology 7 (889): 1-13. 2016

Tomkins, Silvan S. 'Affect theory.' Approaches to emotion. Ed. Klaus Scherer, Paul Ekman. Hillsdale: Laurence Erlbaum (1984),163-195.

Teckenberg-Jansson, Pia, Huotilainen, Minna, Pölkki, Tarja, Lipsanen, Jari, and Järvenpää, Anna-Liisa. 'Rapid effects of neonatal music therapy combined with kangaroo care on prematurely-born infants.' Nordic Journal of Music Therapy, 20.1 (2011): 22-42.

Varga, Somogy. 'Interaction and extended cognition.' Synthese, forthcoming. doi: 10.1007/s11229-015-0861-7.

Varga, Somogy, and Krueger, Joel (2013). 'Background emotions, proximity and distributed emotion regulation. Review of Philosophy and Psychology, 4.2 (2013): 271-92.

von Scheve, C. The Social Calibration of Emotion Expression: An Affective Basis of Microsocial Order. Sociological Theory, 30.1 (2012): 1-14.

von Scheve, Christian, and Ismer, Sven. 'Towards a theory of collective emotions.' Emotion Review, 5.4 (2013): 406-413.

von Scheve, Christian, and Salmela, Mikko (Eds.). Collective Emotions. Perspectives from Psychology, and Sociology. Oxford: Oxford UP, 2014.

Yzerbyt, Vincent, Dumont, Muriel, Wigboldus, Daniel, and Gordijn, Ernestine. 'I feel for us: The impact of categorization and identification on emotions and action tendencies.' British Journal of Social Psychology, 42.4 (2003): 533-49. 
Wheeler, Michael. Extended Consciousness: An Interim Report. The Southern Journal of Philosophy, $53 . S 1$ (2015): 155-75.

Windsor, W. Luke, and de Bézenac, Christophe. 'Music and affordances.' Musicae Scientiae, 16.1 (2012): 102-20.

Witek, Maria. ... and I Feel Good!': The Relationship between Body-Movement, Pleasure and Groove in Music. (Doctoral Thesis). University of Oxford, 2013.

Witek, Maria A. G., Eric F. Clarke, Mikkel Wallentin, Morten L. Kringelbach, and Peter Vuust. 'Syncopation, Body-Movement and Pleasure in Groove Music.' PloS One 9.4 (2014): e94446.

Ward, Dave. 'Enjoying the Spread: Conscious Externalism Reconsidered.' Mind, 121.483 (2012): 731-51.

Wilutzky, Wendy. 'Emotions as Pragmatic and Epistemic Actions.' Frontiers in Psychology, 6.1593 (2015): 1-10.

Zachar, Peter. Psychological Concepts and Biological Psychiatry: A Pbilosophical Analysis. Amsterdam: John Benjamins, 2000.

Zahavi, Dan. 'You, Me and We: The Sharing of Emotional Experiences.' Journal of Consciousness Studies, 22.1-2 (2015): 84-101.

Zajonc, Robert B. 'Emotion and facial efference: A theory reclaimed.' Science, 228.4695 (1985): 15-21. 